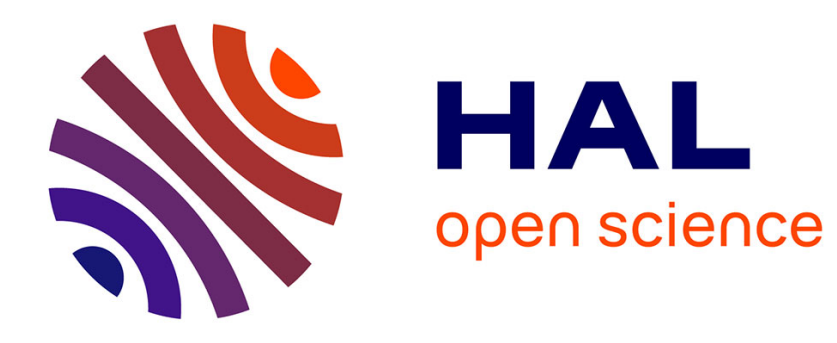

\title{
Ruin probabilities for a regenerative Poisson gap generated risk process
}

Søren Asmussen, Romain Biard

\section{To cite this version:}

Søren Asmussen, Romain Biard. Ruin probabilities for a regenerative Poisson gap generated risk process. European Actuarial Journal, 2011, 1 (1), pp.3-22. 10.1007/s13385-011-0002-8 . hal-00569254v2

\section{HAL Id: hal-00569254 \\ https://hal.science/hal-00569254v2}

Submitted on 22 Apr 2011

HAL is a multi-disciplinary open access archive for the deposit and dissemination of scientific research documents, whether they are published or not. The documents may come from teaching and research institutions in France or abroad, or from public or private research centers.
L'archive ouverte pluridisciplinaire HAL, est destinée au dépôt et à la diffusion de documents scientifiques de niveau recherche, publiés ou non, émanant des établissements d'enseignement et de recherche français ou étrangers, des laboratoires publics ou privés. 


\title{
Ruin probabilities for a regenerative Poisson gap generated risk process Søren Asmussen Romain Biard asmus@imf.au.dkｒbiard@gmail.com \\ Department of Mathematical Sciences, Aarhus University Ny Munkegade, DK-8000 Aarhus C, Denmark
}

\begin{abstract}
A risk process with constant premium rate $c$ and Poisson arrivals of claims is considered. A threshold $r$ is defined for claim interarrival times, such that if $k$ consecutive interarrival times are larger than $r$, then the next claim has distribution $G$. Otherwise, the claim size distribution is $F$. Asymptotic expressions for the infinite horizon ruin probabilities are given for both light- and the heavy-tailed cases. A basic observation is that the process regenerates at each $G$-claim. Also an approach via Markov additive processes is outlined, and heuristics are given for the distribution of the time to ruin.
\end{abstract}

Keywords: Ruin theory; Subexponential distribution; Large deviations; Markov additive process; Finite horizon ruin

\section{Introduction}

In a recent paper, Biard, Lefevre, Loisel \& Nagaraja [9] considered risk processes with constant premium inflow at rate $c$, claims arriving according to a Poisson $(\lambda)$ process $N$ and a certain dependence between claim interarrival times and claims. That is, the claim surplus process $S$ ([5, p. 1]) is given by

$$
S(t)=\sum_{i=1}^{N(t)} U_{i}-c t
$$

where a claim $U_{i}$ arriving at time $t$ has a distribution depending on $\{N(s)\}_{s \leq t}$.

In one model of [9], a threshold $r$ is defined for claim interarrival times, such that if $k$ consecutive interarrival times are larger than $r$, then the claim has distribution $G$. Otherwise, the claim size distribution is $F$. A motivating example in [9] is situations where claims are generated by earthquakes. More precisely, a 'claim' is to be understood as the aggregation of the many individual claims caused by the earthquake. The size of a claim is then positively correlated to the size of the earthquake, and one expects an earthquake to be the more severe the fewer earthquakes have recently occurred so that a $G$-claim should be larger than an $F$-claim in some appropriate sense (see Section 8 for some 
more detailed motivating discussion). Another model in [9] has flooding as a motivating example. Here a close succession of claims is expected to be followed by a more important catastrophe. For more details on the model, see [9] and Section 8. We focus here on the first model, since the treatment of the second is largely very similar (see again the discussion in Section 8). We also take $k=2$ for simplicity; the needed extensions to $k>2$ are outlined, and note also that even the case $k=1$ is non-trivial (but of course simpler than $k \geq 2$ ).

The results of [9] are asymptotic relations for finite horizon ruin probabilities $\psi(u, T)$ when $u$ is large and $T$ is fixed. The present paper complements these results by giving the asymptotics for the infinite horizon ruin probability $\psi(u)$ with initial reserve $u$ as $u \rightarrow \infty$. We also heuristically look into the time $\tau(u)$ of ruin, identify the most likely deterministic time when ruin occurs, which leads to expansions for the finite horizon ruin probability $\psi(u, T)$ when both $u$ and $T$ are large. Here

$\tau(u)=\inf \{t>0: S(t)>u\}, \psi(u)=\mathbb{P}(\tau(u)<\infty), \psi(u, T)=\mathbb{P}(\tau(u) \leq T)$.

Whereas these topics are more mainstream ruin theory than [9], we also need a restriction: it is assumed that all claim sizes are independent. Note, however, that some of the dependence modeling in [9] does not easily generalize to an infinite horizon setting.

Our basic point of view is that of regenerative processes, [4, Ch. VI]. The process regenerates at a $G$-claim in the sense that the process forgets about the preceding large claims. Before the next $G$-claim can occur, it has to wait until $k$ long (short) interarrival times have occured. See Section 2 for formalities. Asmussen, Schmidli \& Schmidt [7] used such a structure to get ruin probability asymptotics in some non-standard risk processes with heavy tails (regeneration is also used in Foss \& Zachary [16] and in Schmidli [21] but in [21] at process levels rather than time instants).

The regenerative set-up is presented in Section 2, with a key calculation (of the mean cycle length) given in Section 3. The first application to the present model is presented in Section 4, where we show that the classical heavytailed asymptotics for the ruin probability $\psi(u)$ as $u \rightarrow \infty$ holds here as well. Section 5 deals with light tails. Using large deviations techniques from Glynn \& Whitt [17] and Duffield \& O'Connell [12], we show that the first order decay of $\psi(u)$ is exponential and we identify the rate $\gamma$ (the adjustment coefficient). Also here the details use the regenerative structure. Section 6 presents an alternative point of view, to view the process as a Markov-modulated risk process where the modulating process is the relevant information on the last two interarrival intervals. This point of view, though not fully exploited here, has the potential of giving stronger results than the large deviations approach, and we demonstrate this by deriving a proper Cramér-Lundberg approximation $\psi(u) \sim C \mathrm{e}^{-\gamma u}$. In Section 7, we then (in part heuristically) derive the conditional distribution of the ruin time given that ruin occurs. Finally Section 8 contains a number of concluding remarks, and in Appendices A-D, we give the details of some of the longer technical calculations.

\section{Preliminaries}

We recall the basic regenerative set-up of [7]: 
Assumption 2.1 There exist random times $\omega_{0}=0, \omega_{1}, \omega_{2}, \ldots$ such that the post- $\omega_{k}$ process $\left\{S\left(t+\omega_{k}\right)-S\left(\omega_{k}\right)\right\}_{t \geq 0}$ is independent of the pre- $\omega_{k}$ process $\{S(t)\}_{0 \leq t \leq \omega_{k}}$ and its distribution does not depend on $k$.

In our setting, $\omega_{1}, \omega_{2}, \ldots$ are the times of $G$-claims. Except for independence of $\left\{S\left(t+\omega_{1}\right)-S\left(\omega_{1}\right)\right\}_{t \geq 0}$, we will typically not need strong conditions on the distribution of the initial cycle $\{S(t)\}_{0 \leq t<\omega_{1}}$. However, some weak technical conditions are given in the respective theorems. When $\omega_{1}=0$, we write $\mathbb{P}_{0}, \mathbb{E}_{0}$. We often use notation like $\mathbb{P}(\omega \leq x)$ instead of $\mathbb{P}_{0}\left(\omega_{2} \leq x\right)$. Define further

$$
M_{k}^{*}=\sup _{\omega_{k} \leq t<\omega_{k+1}}\left(S(t)-S\left(\omega_{k}\right)\right), \quad S_{k}^{*}=S\left(\omega_{k+1}\right), \quad m^{*}=\left|\mathbb{E}_{0} S_{1}^{*}\right|=|\mathbb{E} S(\omega)| .
$$

Under Assumption 2.1, $M_{1}, M_{2}, \ldots$ are i.i.d. and the $S_{k}^{*}-S_{1}^{*}$ form a random walk. A basic assumption of [7] is (note that trivially $\mathbb{P}_{0}\left(S_{1}^{*}>x\right) \leq \mathbb{P}_{0}\left(M_{1}^{*}>\right.$ $x))$ :

Assumption $2.2 \mathbb{P}_{0}\left(S_{1}^{*}>x\right) \sim \mathbb{P}_{0}\left(M_{1}^{*}>x\right), x \rightarrow \infty$.

Then ([7]; for background on subexponential distributions, see, e.g., [13], [5, X.1] or [14]):

Theorem 2.3 Assume in addition to Assumptions 2.1 and 2.2 that $\mathbb{E}_{0} S_{1}^{*}<0$, that both $\bar{H}(x)=\mathbb{P}_{0}\left(S_{1}^{*}>x\right)$ and the integrated tail $\bar{H}_{I}(x)=\int_{x}^{\infty} \bar{H}(y) \mathrm{d} y$ are subexponential tails, and that $\mathbb{P}\left(M_{0}^{*}>x\right)=\mathrm{o}\left(\bar{H}_{I}(x)\right)$. Then $\psi(u) \sim \frac{1}{m^{*}} \bar{H}_{I}(u)$, $u \rightarrow \infty$.

\section{The mean cycle length}

As usual when applying regenerative processes, a main step is computation of the mean cycle length $\mu=\mathbb{E} \omega=\mathbb{E}_{0} \omega_{2}$. To this end, let $V$ denote the generic claim interarrival time and define

$$
\begin{aligned}
p_{+} & =\mathbb{P}(V>r)=\mathrm{e}^{-\lambda r}, \quad p_{-}=\mathbb{P}(V \leq r)=1-\mathrm{e}^{-\lambda r}, \\
m_{+} & =\mathbb{E}[V \mid V>r]=\frac{1}{p_{+}} \int_{r}^{\infty} x \lambda \mathrm{e}^{-\lambda x} \mathrm{~d} x=r+\frac{1}{\lambda}, \\
m_{-} & =\mathbb{E}[V \mid V \leq r]=\frac{1}{p_{-}} \int_{0}^{r} x \lambda \mathrm{e}^{-\lambda x} \mathrm{~d} x=\frac{1}{\lambda}-\frac{r \mathrm{e}^{-\lambda r}}{1-\mathrm{e}^{-\lambda r}} .
\end{aligned}
$$

Recall that a cycle starts just after a $G$-claim and ends at the next. A cycle is initiated by $K$ short claims where $K$ is geometric, $\mathbb{P}(K=k)=p_{-}{ }^{k} p_{+}$; this part $\omega^{(1)}$ of the cycle has mean $\left(p_{-} / p_{+}\right) m_{-}$. After that comes a long part $\omega^{(2)}$ with mean $m_{+}$. For the final part $\omega^{(3)}$, two possibilities occur: The next interarrival time is long and the cycle terminates. This gives an additional contribution to the mean of $p_{+} m_{+}$. The second possibility is that the next interarrival time is short and the cycle starts afresh after that. Putting things together gives

$$
\mu=\frac{p_{-}}{p_{+}} m_{-}+m_{+}+p_{+} m_{+}+p_{-}\left(m_{-}+\mu\right)
$$

so that we have shown 
Theorem 3.1 The mean cycle length $\mu=\mathbb{E} \omega=\mathbb{E}_{0} \omega_{2}$ is given by

$$
\begin{aligned}
\mu & =\frac{1}{p_{+}}\left[\frac{p_{-}}{p_{+}} m_{-}+m_{+}+p_{+} m_{+}+p_{-} m_{-}\right] \\
& =\frac{1}{p_{+}}\left[\left(1+\frac{1}{p_{+}}\right)\left(p_{+} m_{+}+p_{-} m_{-}\right)\right]=\frac{1}{\lambda} \frac{1+p_{+}}{p_{+}{ }^{2}}=\frac{1}{\lambda} \mathrm{e}^{\lambda r}\left(1+\mathrm{e}^{\lambda r}\right) .
\end{aligned}
$$

\section{Heavy tails and regeneration}

Having computed $\mu$, the criterion for stability follows easily. Let $m_{F}, m_{G}$ denote the means of $F$, resp. $G$.

Corollary 4.1 Define $\eta=1-\frac{(\lambda \mu-1) m_{F}+m_{G}}{c \mu}$. If $\eta>0$, then $\psi(u)<1$ for all $u$, whereas $\psi(u) \equiv 1$ if $\eta \leq 0$.

Proof. Let $M$ denote the number of claims in a cycle. By regenerative process theory, $\lambda=\mathbb{E} M / \mu$ and $\lim _{t \rightarrow \infty} S(t) / t$ exists and equals $\mathbb{E} S(\omega) / \mu$. But

$$
\mathbb{E} S(\omega)=\mathbb{E}(M-1) m_{F}+m_{G}-c \mu=(\lambda \mu-1) m_{F}+m_{G}-c \mu .
$$

Thus $\lim S(t) / t$ is $<0$ for $\eta>0$ and $>0$ for $\eta<0$, which immediately gives the conclusion. If $\eta=0$, we need in addition to remark that $S\left(\omega_{1}\right), S\left(\omega_{2}\right), \ldots$ is a mean zero random walk. Hence $\lim \sup _{k} S\left(\omega_{k}\right)=\infty$ so that $\tau(u)<\infty$ for all $u$.

Note that, as the proof reveals, $\eta$ plays the role of the safety loading (the relative amount by which the premium rate $c$ exceeds the expected inflow of claims), cf. $[5, \mathrm{p} .3]$. In the following, it is throughout assumed that $\eta>0$.

We now turn attention to our main result for the heavy-tailed case. We will not necessarily make the assumption, founded in the earthquake interpretation, that $G$ has a heavier tail than $F$ but instead:

Assumption 4.2 There exists a distribution $K$ on $[0, \infty)$ such that both $K$ and the integrated tail $K_{I}$ are subexponential, and that

$$
\bar{F}(x) \sim c_{F} \bar{K}(x), \quad \bar{G}(x) \sim c_{G} \bar{K}(x), \quad x \rightarrow \infty,
$$

where $c_{F}+c_{G}>0$.

The easy consequence of Assumption 4.2 is

Proposition 4.3 Let $A=\sum_{1}^{M} U_{i}$ denote the total accumulated claims in a cycle. Then $\mathbb{P}(A>x) \sim d \bar{K}(x)$, where $d=\mathbb{E}(M-1) c_{F}+c_{G}$.

Proof. By general results on sums of a light-tailed number of subexponential r.v.'s $\left([5\right.$, p. 302] $)$, we have $\mathbb{P}\left(\sum_{1}^{M-1} U_{i}>x\right) \sim \mathbb{E}(M-1) c_{F} \bar{K}(x)$. Since $U_{M}$ has tail $c_{G} \bar{K}(x)$ and is independent of $U_{1}, \ldots, U_{M-1}$, a general convolution result ([5, pp.297-8]) completes the proof.

From e.g. [14], subexponentiality of a distribution $L$ implies that there exists a non-decreasing function $\delta$ with $\delta(x) \rightarrow \infty$ as $x \rightarrow \infty$, such that

$$
\bar{L}(x \pm \delta(x)) \sim \bar{L}(x) \quad \text { as } x \rightarrow \infty .
$$


Lemma 4.4 Let $A \geq 0$ be a r.v. with subexponential distribution $L$ and $\omega \geq 0$ a (not necessarily independent) light-tailed r.v.. Then $\mathbb{P}(A-\omega>x) \sim \bar{L}(x)$ provided $\delta(\cdot)$ in $(4.2)$ can be chosen with $\mathrm{e}^{-g \delta(x)}=\mathrm{o}(\bar{L}(x))$ for all $g>0$.

Proof. Write

$$
\mathbb{P}(A-\omega>x)=\mathbb{P}(A-\omega>x, \omega \leq \delta(x))+\mathbb{P}(A-\omega>x, \omega>\delta(x)) .
$$

Since $\omega$ has finite exponential moments, we have, for some constant $\gamma>0$,

$$
\mathbb{P}(A-\omega>x, \omega>\delta(x)) \leq \mathbb{P}(\omega>\delta(x)) \leq \mathrm{e}^{-\gamma \delta(x)}=\mathrm{o}(\bar{L}(x)) .
$$

Moreover,

$$
\begin{aligned}
\mathbb{P}(A-\omega>x, \omega \leq \delta(x)) & \leq \mathbb{P}(A>x)=\bar{L}(x), \\
\mathbb{P}(A-\omega>x, \omega \leq \delta(x)) & \geq \mathbb{P}(A-\delta(x)>x, \omega \leq \delta(x)) \\
& =\mathbb{P}(A-\delta(x)>x)-\mathbb{P}(A-\delta(x)>x, \omega>\delta(x)) \\
& \sim \bar{L}(x)-\mathrm{o}(\bar{L}(x)) .
\end{aligned}
$$

Putting these estimates together completes the proof.

Here is then our main result on the heavy-tailed case:

Theorem 4.5 Assume in addition to Assumption 4.2 that $\mathrm{e}^{-g \delta(x)}=\mathrm{o}\left(\bar{K}_{I}(x)\right)$ for all $g>0$, and that $\mathbb{P}\left(M_{0}^{*}>x\right)=\mathrm{o}\left(\bar{G}_{I}(x)\right)$. Then

$$
\psi(u) \sim \frac{d}{\eta c \mu} \bar{L}_{I}(u), \quad u \rightarrow \infty .
$$

Proof. Define $S^{*}=S(\omega), M^{*}=\sup _{t \leq \omega} S(t)$. Then

$$
\begin{aligned}
\mathbb{P}(A>x) & \sim d \bar{K}(x), \\
\mathbb{P}\left(S^{*}>x\right) & \sim d \bar{K}(x), \\
\mathbb{P}\left(M^{*}>x\right) & \sim d \bar{K}(x)
\end{aligned}
$$

as $x \rightarrow \infty$, from which the result follows from Theorem 3.1, using (4.1) to identify the constant $|\mathbb{E} S(\omega)|$ in front of $d \bar{K}(u)$. Indeed, (4.5) is just Proposition 4.3, (4.6) then follows from $S^{*}=A-c \omega$ and Lemma 4.4, and (4.6) is then a consequence of $S^{*} \leq M^{*} \leq A$.

Remark 4.6 The condition $\mathrm{e}^{-g \delta(x)}=\mathrm{o}(\bar{L}(x))$ for all $x$ holds for $L$ regularly varying (take $h(x)=f \log x$ with $f$ sufficiently large), the lognormal distribution $\left(h(x)=x / \log ^{2} x\right)$ and the heavy-tailed Weibull with $\bar{L}(x)=\mathrm{e}^{-x^{\beta}}$ with $\beta<1 / 2$ $\left(h(x)=x^{1-\beta^{*}}\right.$ with $\left.\beta<\beta^{*}<1\right)$. Thus, the condition covers most standard heavy-tailed distributions except the ones closest to the light-tailed case. Since with independent $A, \omega$ with $A$ subexponential, $A$ and $A-\omega$ always have the same tail ([5, p. 306]), one could believe that the condition is just technical. However, in Appendix D we show it is not.

However, whereas the approach taken here departs from the general discussion in Lemma 4.4, we can in fact prove Theorem 4.5 without the condition by using the special structure for our problem. We have taken here the point of view that the general discussion is of more interest. 


\section{$5 \quad$ Light tails and regeneration}

We now assume both $G$ and $F$ are light-tailed and write $\widehat{F}[\cdot]$ for the m.g.f. of $F$ and $\widehat{G}[\cdot]$ for the m.g.f. of $G$. To obtain the first order asymptotics of the ruin probability $\psi(u)$ as $u \rightarrow \infty$, the theory of large deviations ([5, XIII.1]) suggests that we first establish that in a suitable $\alpha$-range,

$$
\frac{1}{t} \log \mathbb{E} \mathrm{e}^{\alpha S(t)} \rightarrow \kappa(\alpha)
$$

for some function $\kappa$. If $\kappa(\gamma)=0$ for some $\gamma>0$, it then holds under weak additional conditions that $\psi(u)$ is of rough order $\mathrm{e}^{-\gamma u}$ in the sense that

$$
\frac{1}{u} \log \psi(u) \rightarrow-\gamma
$$

The precise additional conditions are in Glynn \& Whitt [17] and Duffield \& O'Connell [12]. Note that the set-up of [17] is discrete time, whereas we here, as in [12], have a continuous-time problem. The conditions of [12] are somewhat more technical to verify than those of [17], but luckily, the path structure (1.1) essentially reduces the problem to discrete time; see Section 7.2 for details. Thus, we will omit the easy verification of conditions of [17] beyond (5.1) and the existence of $\gamma$.

We proceed in two steps, of which the first is:

Proposition $5.1 \varphi(\alpha, \beta)=\mathbb{E}\left[\mathrm{e}^{\alpha S(\omega)} \mathrm{e}^{\beta \omega}\right]$ is given by

$$
\varphi(\alpha, \beta)=\frac{g(\alpha, \beta)^{2} \widehat{F}[\alpha] \widehat{G}[\alpha]}{1-k(\alpha, \beta) \widehat{F}[\alpha][1+g(\alpha, \beta) \widehat{F}[\alpha]]},
$$

where

$$
\begin{aligned}
g(\alpha, \beta) & =\frac{\lambda}{\lambda+c \alpha-\beta} \mathrm{e}^{(\beta-c \alpha-\lambda) r} \\
k(\alpha, \beta) & =\frac{\lambda}{\lambda+c \alpha-\beta}\left[1-\mathrm{e}^{(\beta-c \alpha-\lambda) r}\right] .
\end{aligned}
$$

Proof. The argument is similar to the one for (3.4), though more elaborate. We will need the formulas

$$
\begin{aligned}
& \widehat{A}^{+}[\alpha]=\mathbb{E}\left[\mathrm{e}^{\alpha V} \mid V>r\right]=\frac{\lambda}{\lambda-\alpha} \mathrm{e}^{\alpha r} \\
& \widehat{A}^{-}[\alpha]=\mathbb{E}\left[\mathrm{e}^{\alpha V} \mid V \leq r\right]=\frac{\lambda}{\lambda-\alpha}\left(\frac{1-\mathrm{e}^{-(\lambda-\alpha) r}}{1-\mathrm{e}^{-\lambda r}}\right) .
\end{aligned}
$$

Let $S_{1}^{\#}=S\left(\omega^{(1)}\right), S_{2}^{\#}=S\left(\omega^{(1)}+\omega^{(2)}\right)-S\left(\omega^{(1)}\right), S_{3}^{\#}=S\left(\omega^{(1)}+\omega^{(2)}+\omega^{(3)}\right)-$ $S\left(\omega^{(1)}+\omega^{(2)}\right)$ with $\omega^{(1)}, \omega^{(2)}, \omega^{(3)}$ as in Section 3. Then

$$
\varphi(\alpha, \beta)=\prod_{i=1}^{3} \mathbb{E}\left[\mathrm{e}^{\alpha S_{i}^{\#}} \mathrm{e}^{\beta \omega^{(i)}}\right] .
$$

The easy case is $i=2$ where we can write $S_{2}^{\#}=U_{F}-c V^{+}, \omega^{(2)}=V^{+}$where $U_{F}$ has distribution $F$ and $V^{+}$the conditional distribution of $V$ given $V>r$. This gives

$$
\mathbb{E}\left[\mathrm{e}^{\alpha S_{2}^{\#}} \mathrm{e}^{\beta \omega^{(2)}}\right]=\widehat{F}[\alpha] \widehat{A}^{+}[\beta-c \alpha]
$$


For $i=3$, considering the same two possibilities as for the mean gives by a similar sample path representation the expression

$$
p_{+} \widehat{G}[\alpha] \widehat{A}^{+}[\beta-c \alpha]+p_{-} \widehat{A}^{-}[\beta-c \alpha] \widehat{F}[\alpha] \varphi(\alpha, \beta)
$$

for $\mathbb{E}\left[\mathrm{e}^{\alpha S_{3}^{\#}} \mathrm{e}^{\beta \omega^{(3)}}\right]$. Finally, $\mathbb{E}\left[\mathrm{e}^{\alpha S_{1}^{\#}} \mathrm{e}^{\beta \omega^{(1)}}\right]$ is given by

$$
\mathbb{E}\left[\widehat{F}[\alpha]^{K} \widehat{A}^{-}[\beta-c \alpha]^{K}\right]=\frac{p_{+}}{1-p_{-} \widehat{F}[\alpha] \widehat{A}^{-}[\beta-c \alpha]} .
$$

Putting things together proves the proposition.

To obtain (5.1) from (5.3), we use renewal theory. Let $Z(t)=\mathbb{E}_{0} \mathrm{e}^{\alpha S(t)}$. Conditioning on $\omega$ then yields the renewal equation

$$
Z(t)=\mathbb{E}\left[\mathrm{e}^{\alpha S(t)} ; \omega>t\right]+\int_{0}^{t} Z(t-y) \mathbb{E}\left[\mathrm{e}^{\alpha S(\omega)} ; \omega \in \mathrm{d} y\right]
$$

Choose $\kappa=\kappa(\alpha)$ as the solution of

$$
1=\varphi(\alpha, \kappa)=\int_{0}^{\infty} \mathrm{e}^{-\kappa y} \mathbb{E}\left[\mathrm{e}^{\alpha S(\omega)} ; \omega \in \mathrm{d} y\right] .
$$

Then by standard renewal theory, $\mathrm{e}^{-\kappa(\alpha) t} Z(t)$ has limit

$$
\int_{0}^{\infty} \mathrm{e}^{-\kappa(\alpha) v} \mathbb{E}\left[\mathrm{e}^{\alpha S(v)} ; \omega>v\right] \mathrm{d} v / \int_{0}^{\infty} v \mathrm{e}^{-\kappa(\alpha) v} \mathbb{E}\left[\mathrm{e}^{\alpha S(v)} ; \omega \in \mathrm{d} v\right]
$$

as $t \rightarrow \infty$. This implies (5.1). Taking $\gamma>0$ as solution of $\kappa(\gamma)=0$ gives the ruin probability approximation (5.2) in the zero-delayed case. Putting things together and involving the further technical conditions of [17] gives:

Theorem 5.2 Assume there exists $\gamma>0$ such that (5.1) holds in a neighborhood of $\gamma$, that $\kappa(\gamma)=0$ and that $\mathbb{P}\left(M_{0}^{*}>x\right)=\mathrm{o}\left(\mathrm{e}^{-\gamma x}\right)$. Then (5.2) holds.

\section{The Markov additive point of view}

The large deviations approach of Section 5 is quick and applicable to more general models, but also somewhat imprecise by only giving logarithmic asymptotics. In this section, we outline how a Markov additive point of view ([5, III.4, VI.3b, VII, IX.5] can lead to more precise asymptotics, in particular a Cramér-Lundberg approximation of the form $\psi(u) \sim C \mathrm{e}^{-\gamma u}$.

A special case of a Markov additive model for the claim surplus process $S$ is the Markov-modulated Cramér-Lundberg model. It asserts that there is a driving background Markov process $J$ with state space $E$, such that the claim arrival intensity is $\lambda_{i}$ when $J(t)=i$ and the claim size distribution is $F_{i}$. Cf. [5, III.4, Ch. VII]. This covers the model of the present paper as will next be explained.

The relevant background information for our Poisson gap generated risk process at time $t$ is at a first sight $(b, a)$ where $a$ is the time since the last arrival and $b$ is the length of the previous interarrival interval. However, this description is somewhat redundant since what matters is really comparisons of 
$b, a$ to the threshold length $r$. Accordingly, we define $J(t)=-, a$ if $b, a \leq r$, $J(t)=-,+$ if $b \leq r, a>r, J(t)=+, a$ if $b>r, a \leq r$ and $J(t)=+,+$ if $a, b>r$. Thus, the state space of $J$ is

$$
E=\{-\} \times[0, r] \cup\{-,+\} \cup\{+\} \times[0, r] \cup\{+,+\}
$$

and $\lambda_{i} \equiv \lambda, F_{i}=G$ when $i=+,+, F_{i}=F$ for all other $i \in E$. Note that after a $G$-claim, $J$ is reset to -, 0 . An important point is that $E$ is uncountable, which presents a complication compared to the most studied finite case, cf. [5, Ch. VII].

For the analysis, we proceed according to the program outlined for the infinite case in [5, Remark III.4.8 p. 58] (the examples treated in [5] are periodic environment in VII.6 and the renewal risk processes in VI.3b). For a fixed $\theta$, we need to determine $\kappa(\theta)$ and $h^{\theta}: E \rightarrow(0, \infty)$ such that

$$
\mathbb{E}_{i} \mathrm{e}^{\theta S(t)} \sim \mathrm{e}^{t \kappa(\theta)} h^{\theta}(i), \quad t \rightarrow \infty,
$$

where $\mathbb{E}_{i}$ refers to the case $J(0)=i$. Noting that $J(0)=-, 0$ corresponds to the zero-delayed regenerative case, we know from Section 5 that this holds for $i=-, 0$ with the same $\kappa(\theta)$ as computed there and $h^{\theta}(-, 0)$ given by (5.8). For a general $i$, we have

$$
\mathbb{E}_{i} \mathrm{e}^{\theta S(t)}=\int_{0}^{t} \mathbb{E}_{i}\left[\mathrm{e}^{\theta S\left(\omega_{1}\right)} ; \omega_{1} \in \mathrm{d} s\right] \mathbb{E}_{-, 0} \mathrm{e}^{\theta S(t-s)}+\mathbb{E}_{i}\left[\mathrm{e}^{\theta S(t)} ; \omega_{1}>t\right] .
$$

Letting $t \rightarrow \infty$, the second term vanishes, and using (6.1) with $i=-, 0$ gives (6.1) for a general $i$ with

$$
h^{\theta}(i)=\mathbb{E}_{i} \mathrm{e}^{\theta S\left(\omega_{1}\right)-\kappa(\theta) \omega_{1}} .
$$

Here, as will be shown in Appendix C, we have with $g, h$ as in Proposition 5.1 and with

$$
\begin{aligned}
g^{(a)}(\alpha, \beta) & =\frac{\lambda}{\lambda+c \alpha-\beta} \mathrm{e}^{(\beta-c \alpha-\lambda)(r-a)}, \\
k^{(a)}(\alpha, \beta) & =\frac{\lambda}{\lambda+c \alpha-\beta}\left(1-\mathrm{e}^{(\beta-c \alpha-\lambda)(r-a)}\right),
\end{aligned}
$$

that

$$
\begin{aligned}
h^{\theta}(-, 0) & =\frac{g(\theta,-\kappa(\theta))^{2} \widehat{F}[\theta] \widehat{G}[\theta]}{1-k(\theta,-\kappa(\theta)) \widehat{F}[\theta](1+g(\theta,-\kappa(\theta)) \widehat{F}[\theta])} \\
h^{\theta}(+,+) & =\frac{\lambda}{\lambda+c \theta+\kappa(\theta)} \widehat{G}[\theta] \\
h^{\theta}(+, a)= & \frac{k^{(a)}(\theta,-\kappa(\theta)) g(\theta,-\kappa(\theta))^{2} \widehat{F}[\theta]^{2} \widehat{G}[\theta]}{1-k(\theta,-\kappa(\theta)) \widehat{F}[\theta](1+g(\theta,-\kappa(\theta)) \widehat{F}[\theta])}+g^{(a)}(\theta,-\kappa(\theta)) \widehat{G}[\theta] \\
h^{\theta}(-,+)= & \frac{\lambda}{\lambda+c \theta+\kappa(\theta)} \frac{g(\theta,-\kappa(\theta)) \widehat{F}[\theta] \widehat{G}[\theta](1-k(\theta,-\kappa(\theta)) \widehat{F}[\theta])}{1-k(\theta,-\kappa(\theta)) \widehat{F}[\theta](1+g(\theta,-\kappa(\theta)) \widehat{F}[\theta])} \\
h^{\theta}(-, a)= & \frac{g(\theta,-\kappa(\theta)) \widehat{F}[\theta] \widehat{G}[\theta]}{1-k(\theta,-\kappa(\theta)) \widehat{F}[\theta](1+g(\theta,-\kappa(\theta)) \widehat{F}[\theta])}\left\{k^{(a)}(\theta,-\kappa(\theta)) g(\theta,-\kappa(\theta)) \widehat{F}[\theta]\right. \\
& \left.+g^{(a)}(\theta,-\kappa(\theta)) \widehat{G}[\theta](1-k(\theta,-\kappa(\theta)) \widehat{F}[\theta])\right\}
\end{aligned}
$$


From [5, Remark III.4.8 p. 58], we now have the representation

$$
\psi_{i}(u)=\mathrm{e}^{-\gamma u} h^{\gamma}(i) \widetilde{\mathbb{E}}_{i}\left[\mathrm{e}^{-\gamma \xi(u)} / h^{\gamma}(J(\tau(u)))\right],
$$

where $\psi_{i}(u)=\mathbb{P}_{i}(\tau(u)<\infty), \xi(u)=S(\tau(u))-u$ and $\widetilde{\mathbb{E}}$ refers to a suitably exponentially tilted probability measure. Now the process $\{(\xi(u), J(\tau(u)))\}_{u>0}$ is regenerative (say, regeneration occurs at $u$-values with $\xi(u)=0, J(\tau(u))=$ $+,+)$. Hence a limit $\left(\xi(\infty), J^{*}(\infty)\right)$ in distribution exists and is independent of $i$, and (6.8) gives:

Theorem 6.1 In the light-tailed case,

$$
\psi_{i}(u) \sim \mathrm{e}^{-\gamma u} h^{\gamma}(i) C,
$$

where $C=\mathbb{E}\left[\mathrm{e}^{-\gamma \xi(\infty)} / h^{\gamma}\left(J^{*}(\infty)\right)\right]$.

We note that whereas $h^{\gamma}(i)$ is explicitly computable for all $i$, this is hardly the case for $C$ (even in the finite case, the evaluation leads into ladder height problems with no explicit solution). The Markov additive point of view in the heavy-tailed case is exploited in Foss \& Zachary [16], and in fact, Theorem 4.5 can be derived from that paper. However, the details are hardly shorter than the present proof via [7].

\section{The time to ruin}

Let $\mathbb{P}^{(u)}$ denote the conditional distribution given $\tau(u)<\infty$. Our goal here is to determine the asymptotic form of the $\mathbb{P}^{(u)}$-distribution of the ruin time $\tau(u)$. Once this has been obtained, asymptotic expansions of the finite horizon ruin probabilities follow from

$$
\psi(u, T)=\psi(u) \mathbb{P}^{(u)}(\tau(u) \leq T),
$$

but we shall not spell out the easy details.

\subsection{The heavy-tailed case}

We here state a result that we strongly believe to be true, with a sketch (but only sketch!) of proof. Results of this type have their origin in Asmussen \& Klüppelberg [6]). The setting is that of the general regenerative structure in Section 2. which, of course, covers our Poisson gap model as a special case . We will need the mean excess function $e(x)$ of $H$ given by $e(x)=\bar{H}_{I}(x) / \bar{H}(x)$. There are two main cases (cf. [5, pp. 300-301]):

(a) $\bar{H}$ is a regularly varying tail with index $\alpha>1$, i.e. $\bar{H}(x)=L(x) / x^{\alpha}$ with $L$ slowly varying at $+\infty$. Then $\bar{H}_{I}(x) \sim L(x) / x^{\alpha-1}(\alpha-1), e(x) \sim x /(\alpha-1)$ and $\bar{H}(x+w e(x)) \sim \bar{H}(x) \mathbb{P}(W>w)$ where $W$ is Pareto with mean 1 and the same $\alpha$, i.e. with tail $(1+w /(\alpha-1))^{-\alpha}$;

(b) $e(x)$ is self-neglecting in the sense that for all $y, e(x+y e(x)) / e(x) \rightarrow 1$ as $x \rightarrow \infty$. Then $\bar{H}(x+w e(x)) \sim \bar{H}(x) \mathbb{P}(W>w)$ where $W$ is standard exponential. 
Note that the self-neglecting property holds in standard examples of subexponential distributions with lighter tails than regularly varying, say the lognormal or the DFR Weibull. For further discussion and background, see [6], [8], [20].

Recall that $m^{*}=|\mathbb{E} S(\omega)|$.

Conjecture 7.1 In addition to the conditions of Theorem 2.3, assume that either (a) or (b) holds and that $\mathbb{E}_{0}\left[\omega_{2} \mid M_{2}^{*}>x\right]=\mathrm{o}(e(x))$. Then $\tau(u) / e(u) \rightarrow$ $W \mu / m^{*}$ in $\mathbb{P}^{(u)}$-distribution

Proof. It is contained in the proof of [7] that $\psi(u) \sim \mathbb{P}\left(M^{*}>u\right)=\mathbb{P}\left(\tau^{*}(u)<\infty\right)$ where $M^{*}=\sup \left(S^{*}, S_{2}^{*}, \ldots\right), \tau^{*}(u)=\inf \left\{k: S_{k}^{*}>u\right\}$. It follows (using more general results by Foss, Palmowski \& Zachary [15] in the first step) that

$$
\begin{aligned}
\mathbb{P}\left(\tau^{*}(u) \leq a e(u)\right) & \sim \sum_{k=1}^{a e(u)} \bar{H}\left(k m^{*}+u\right) \sim \int_{0}^{a e(u)} \bar{H}\left(z m^{*}+u\right) \mathrm{d} z \\
& =\left[\bar{H}_{I}(u)-\bar{H}_{I}\left(u+a m^{*} e(u)\right)\right] / m^{*} \\
& =\left[\bar{H}(u) e(u)-\bar{H}\left(u+a m^{*} e(u)\right) e\left(u+a m^{*} e(u)\right)\right] / m^{*}(7.1)
\end{aligned}
$$

In case (a), the asymptotics of [.] is

$$
\begin{aligned}
& \frac{L(u)}{u^{\alpha}} \cdot \frac{u}{\alpha-1}-\frac{L\left(u+a m^{*} u /(\alpha-1)\right)}{\left(u+a m^{*} e(u)\right)^{\alpha}} \cdot \frac{u+a m^{*} u /(\alpha-1)}{\alpha-1} \\
\sim & \frac{L(u)}{u^{\alpha}} \cdot \frac{u}{\alpha-1}-\frac{L(u)}{\left(u+a m^{*} u /(\alpha-1)\right)^{\alpha}} \cdot \frac{u+a m^{*} u /(\alpha-1)}{\alpha-1} \\
= & \frac{L(u)}{(\alpha-1) u^{\alpha-1}}\left[1-\frac{1}{\left(1+a m^{*} /(\alpha-1)\right)^{\alpha-1}}\right]=\bar{H}_{I}(u) \mathbb{P}\left(W / m^{*} \leq a\right) .
\end{aligned}
$$

It is further strongly suggested by the proof of [7] that cycles prior to the one with ruin are 'typical' and so should have approximate mean $\mu$. Thus one expects that (recall the assumption $\mathbb{E}_{0}\left[\omega_{2} \mid M_{2}^{*}>x\right]=\mathrm{o}(e(x))$ )

$$
\tau(u) \approx\left(\tau^{*}(u)-1\right) \mu+\mathbb{E}_{0}\left[\omega_{2} \mid M_{2}^{*}>u\right] \approx \tau^{*}(u) \mu
$$

on the set where $\tau(u)<\infty$. Therefore, by Theorem 2.3,

$$
\begin{aligned}
\mathbb{P}^{(u)}(\tau(u) / e(u) \leq b) & =\frac{\mathbb{P}(\mu \tau(u) / e(u \leq b)}{\psi(u)} \\
& \approx \frac{\bar{H}_{I}(u) \mathbb{P}\left(\mu W / m^{*} \leq b\right) / m^{*}}{\bar{H}_{I}(u) / m^{*}}=\mathbb{P}\left(\mu W / m^{*} \leq b\right),
\end{aligned}
$$

showing the claim in case (a).

In case (b) the asymptotics of (7.1) is

$$
\left[\bar{H}(u) e(u)-\bar{H}\left(u+a m^{*} e(u)\right) e(u)\right] / m^{*} \sim \bar{H}_{I}(u)\left[1-\mathbb{P}\left(W / m^{*}>a\right)\right] / m^{*} .
$$

Now just proceed as in case (a). 


\subsection{The light-tailed case}

With light tails, we have

Proposition 7.2 For each $\delta>0, \frac{1}{u} \log \mathbb{P}\left(\left|\frac{\tau(u)}{u}-\frac{1}{\kappa^{\prime}(\gamma)}\right| \leq \delta\right) \rightarrow-\gamma$.

Proof. A discrete time version of the results is in Glynn \& Whitt [17] (see also [5, p. 404]), but is less explicit in the continuous-time setting of Duffield \& O'Connell [12]. However, with the path structure (1.1) the continuous-time version is easily derived from the discrete-time one. Let $\tau_{0}(u)=\inf \{n \in \mathbb{N}$ : $S(n)>u\}$. Then clearly $\tau(u) \leq \tau_{0}(u)$, whereas (1.1) implies $\tau_{0}(u-c) \leq \tau(u)+1$. Further, by [17],

$$
\frac{1}{u} \log \mathbb{P}\left(\left|\frac{\tau_{0}(u)}{u}-\frac{1}{\kappa^{\prime}(\gamma)}\right| \leq \delta\right) \rightarrow-\gamma,
$$

which together with (5.2) gives

$$
\frac{1}{u} \log \mathbb{P}\left(\left|\frac{\tau_{0}(u)}{u}-\frac{1}{\kappa^{\prime}(\gamma)}\right|>\delta, \tau_{0}(u)<\infty\right) \rightarrow 0 .
$$

Now write

$$
\begin{aligned}
& \mathbb{P}\left(\left|\frac{\tau(u)}{u}-\frac{1}{\kappa^{\prime}(\gamma)}\right| \leq \delta\right) \\
& \quad=\mathbb{P}\left(\frac{\tau(u)}{u}-\frac{1}{\kappa^{\prime}(\gamma)} \leq \delta\right)-\mathbb{P}\left(\frac{\tau(u)}{u}-\frac{1}{\kappa^{\prime}(\gamma)}<-\delta\right)
\end{aligned}
$$

Recalling $\tau(u) \leq \tau_{0}(u)$, the first term in (7.5) can be rewritten as

$$
\mathbb{P}\left(\frac{\tau_{0}(u)}{u}-\frac{1}{\kappa^{\prime}(\gamma)} \leq \delta\right)+\mathbb{P}\left(\frac{\tau_{0}(u)}{u}-\frac{1}{\kappa^{\prime}(\gamma)}>\delta, \frac{\tau(u)}{u}-\frac{1}{\kappa^{\prime}(\gamma)} \leq \delta\right) .
$$

Using (7.2), (7.3) shows that the first term dominates the second in the logarithmic sense. Similarly, the second term in (7.5) can be rewritten as

$$
\mathbb{P}\left(\frac{\tau_{0}(u)}{u}-\frac{1}{\kappa^{\prime}(\gamma)}<-\delta\right)-\mathbb{P}\left(\frac{\tau_{0}(u)}{u}-\frac{1}{\kappa^{\prime}(\gamma)}>-\delta, \frac{\tau(u)}{u}-\frac{1}{\kappa^{\prime}(\gamma)}<-\delta\right) .
$$

Recalling $\tau_{0}(u-c) \leq \tau(u)+1$, the second term can be bounded by

$$
\begin{aligned}
& \mathbb{P}\left(\frac{\tau_{0}(u-c)-1}{u}-\frac{1}{\kappa^{\prime}(\gamma)} \leq-\delta\right) \\
& \quad=\mathbb{P}\left(\frac{\tau_{0}(u-c)}{u-c}-\frac{1}{\kappa^{\prime}(\gamma)} \leq-\delta \frac{u}{u-c}+\frac{1+c / \kappa^{\prime}(\gamma)}{u-c}\right)
\end{aligned}
$$

which is at most

$$
\mathbb{P}\left(\frac{\tau_{0}(u-c)}{u-c}-\frac{1}{\kappa^{\prime}(\gamma)} \leq-\delta / 2\right)
$$

for large $u$. Collecting terms shows that the logarithmic order of (7.4) is the same as that of

$$
\mathbb{P}\left(\left|\frac{\tau_{0}(u)}{u}-\frac{1}{\kappa^{\prime}(\gamma)}\right| \leq \delta\right)
$$

Using (7.2) completes the proof.

For discussion of Proposition 7.2, see Section 8. 


\section{Remarks and extensions}

1. Of further recent studies of risk models with dependence between claim sizes and interarrival times, we mention in particular Albrecher \& Boxma [2], Boudreault, Cossette, Landriault \& Marceau [10], Asimit \& Badescu [3] and $\mathrm{Li}$, Tang \& Wu [19]. A survey of different types of risk models with dependence and references is in [5, Ch. XIII].

2. One main direction in earthquake modeling is stress release models where for a stochastic process $X, X(t)$ represents the seismic pressure at time $t$. Between eartquakes, $X$ increases (say linearly) and a downward jump (earthquake) occurs (say) at a rate $\lambda(x)$ depending on the current level $X(t)=x$ of the seismic pressure. Clearly, the jump size should be stochastically increasing in $x$, which in turn is increasing in the time since the last jump. This suggests that the size of earthquake should be stochastically increasing in the time since the last earthquake and, more generally, in the next-to-last interarrival times and the previous ones. This thereby also motivates our model, even only as an approximation.

For a general impression of the above type of earthquake modeling, we refer to the section 'Models and statistics in seismology' in the volume [11] (here also statistical justifications can be found).

3. In Biard, Lefevre, Loisel \& Nagaraja [9], a flooding model is also considered which has a regenerative structure as well. The difference between the two models is the cycle structure. In the earthquake model the process regenerates at a $G$-claim, so after a sequence of $k$ successive interarrival times larger than $r$. In the flooding model, the process regenerates after a sequence of at least $k$ successive interarrival times smaller than $r$ followed by one larger than $r$.

To substantiate the claim made in Section 1 that the regenerative analysis carries over to this case, we will as an example derive the mean cycle length for also $k=2$. Let $\omega$ be the cycle length and $\mu$ its mean. A cycle is initiated by $K$ long interarrival times where $K$ is geometric, $\mathbb{P}(K=k)=p_{+}{ }^{k} p_{-}$; this part $\omega^{(1)}$ of the cycle has mean $\left(p_{+} / p_{-}\right) m_{+}$. After that comes a short part $\omega^{(2)}$ with mean $m_{-}$. For the final part $\omega^{(3)}$, two possibilities occur: The next interarrival time is short and the cycle terminates after $K^{\prime}$ short interarrival times where $K^{\prime}$ is geometric plus one other long time. This gives an additional contribution to the mean of $p_{-}\left(m_{-}+\frac{p_{-}}{p_{+}} m_{-}+m_{+}\right)$. The second possibility is that the next interarrival time is long and the cycle starts afresh after that. Putting things together gives

$$
\mu=\frac{p_{+}}{p_{-}} m_{+}+m_{-}+p_{-}\left(m_{-}+\frac{p_{-}}{p_{+}} m_{-}+m_{+}\right)+p_{+}\left(m_{+}+\mu\right)
$$

so that

$$
\begin{aligned}
\mu & =\frac{1}{p_{-}}\left[\frac{p_{+}}{p_{-}} m_{+}+m_{-}+p_{-}\left(m_{-}+\frac{p_{-}}{p_{+}} m_{-}+m_{+}\right)+p_{+} m_{+}\right] \\
& =\frac{1}{p_{-}}\left[\frac{1}{p_{-}} m_{+}+\frac{1}{p_{+}} m_{-}\right]=\frac{1}{p_{+} p_{-}{ }^{2}} \frac{1}{\lambda}=\frac{1}{\lambda} \frac{\mathrm{e}^{3 \lambda r}}{\left(1-\mathrm{e}^{\lambda r}\right)^{2}} .
\end{aligned}
$$


4. In the earthquake interpretation of the model, one could argue that an earthquake triggers not only a single claim but a whole cascade due to the effects of afterquakes. This is conveniently modeled by a shot-noise process with the same characteristics of the primary process of initiation of cascades as in this paper. Many of our conclusions carry over in an easy matter to this case. The basic fact is that replacing cascades by a single claim of the time of initiation changes neither the exact asymptotics in the heavy-tailed case nor the logarithmic asymptotics in the light-tailed case, cf. Albrecher \& Asmussen [1].

5. As the two previous items suggest, the techniques of the paper carry far beyond our specific model, so that in a wider perspective, one could view the paper as a case study in regenerative methods.

6. A different approach to the heavy-tailed case is reduced load equivalence, which is a way to formalize the frequently occuring phenomenon that if a risk process $S^{\prime}$ with heavy tails is perturbed by a process $S^{\prime \prime}$ with drift zero and lighter tails, then the ruin probability asymptotics for $S=S^{\prime}+S^{\prime \prime}$ remains the same as for $S^{\prime}$. See for example Jelenković, Momcilović \& Zwart [18]. In our setting, $S^{\prime \prime}$ consists of the centered $F$-claims,

$$
S^{\prime \prime}(t)=\sum_{i=1}^{N(t)} U_{i}^{F}-\frac{m^{*}-m_{G}}{\mu} t
$$

where $U_{i}^{F}=U_{i}$ if the $i$ th claim is an $F$-claim and $U_{i}^{F}=0$ if it is a $G$ claim, and $S^{\prime}$ is the renewal risk model with interarrival times distributed as $\omega$, claim size distribution $G$, and premium rate $c^{\prime}=m_{G} / \mu$. However, verification of the technical conditions of [18] is messy, and one should also note that the results obtained in this way only cover so-called square-root insensitivity, excluding for example that $\bar{G}(x)=\mathrm{e}^{-x^{\beta}}$ with $1 / 2<\beta<1$.

7. The analysis of Section 4 easily generalizes to the renewal model, where the time between claims is not necessarily exponential but follows a general distribution. Since one of us has repeatedly questioned how worthwhile such a generalization is (e.g. [5, pp. 12,152-153]), we have chosen not to give the details!

8. Proposition 7.2 strongly suggests that, as in many other risk models, the most likely time of ruin is $u / \kappa^{\prime}(\gamma)$. However, because of the logarithmic form the result is not entirely satisfying. For example, it does not exclude that

$$
\mathbb{P}^{(u)}\left(\left|\frac{\tau(u)}{u}-\frac{1}{\kappa^{\prime}(\gamma)}\right| \leq \delta\right) \rightarrow 0 .
$$

Classical theory $([5, \mathrm{~V} .4])$ suggests that

$$
\frac{\tau(u)-u / \kappa^{\prime}(\gamma)}{\left[u \kappa^{\prime \prime}(\gamma) / \kappa^{\prime}(\gamma)^{3}\right]^{1 / 2}}
$$

has a standard normal limit in $\mathbb{P}^{(u)}$-distribution, which shows that the limit in (8.1) is 1 as one would expect. A rigorous verification of this 
CLT could, e.g., proceed via the Markov additive approach but requires several steps which are easy for a finite Markov additive process but at best tedious in the general case. First one needs to identify the evolvement of the process under the $\gamma$-tilted distribution $\widetilde{\mathbb{P}}$, next to derive a CLT for $S$ under $\widetilde{\mathbb{P}}$ and finally to justify an application of Anscombe's theorem together with Stam's lemma.

\section{A Regenerative calculations for $k>2$}

Now, let us prove that for $k \geq 2$,

$$
\mu^{(k)}=\frac{\mathrm{e}^{\lambda r}}{\lambda} \frac{\mathrm{e}^{\lambda k r}-1}{\mathrm{e}^{\lambda r}-1},
$$

where $\mu^{(k)}$ is the mean of the cycle length in the $k$-case, which we denote a $k$-cycle. For $k=2$, we can easily check that

$$
\frac{1}{\lambda} \mathrm{e}^{\lambda r}\left(1+\mathrm{e}^{\lambda r}\right)=\frac{\mathrm{e}^{\lambda r}}{\lambda} \frac{\mathrm{e}^{\lambda 2 r}-1}{\mathrm{e}^{\lambda r}-1} .
$$

We assume now the result holds for some $k \geq 2$. After a $k$-cycle, we have two possibilities. The next interarrival time is long and we get a $(k+1)$-cycle. This gives an additional contribution to the mean of a $(k+1)$-cycle length of $p_{+} m_{+}$. The second possibility is that the next interarrival time is short and the $(k+1)$-cycle starts afresh after that. Putting things together gives

$$
\mu^{(k+1)}=\mu^{(k)}+p_{+} m_{+}+p_{-}\left(m_{-}+\mu^{(k+1)}\right)
$$

so that

$$
\begin{aligned}
\mu^{(k+1)} & =\frac{1}{p_{+}}\left[\mu^{(k)}+p_{+} m_{+}+p_{-} m_{-}\right]=\mathrm{e}^{\lambda r}\left[\frac{\mathrm{e}^{\lambda r}}{\lambda} \frac{\mathrm{e}^{\lambda k r}-1}{\mathrm{e}^{\lambda r}-1}+\frac{1}{\lambda}\right], \\
& =\frac{\mathrm{e}^{\lambda r}}{\lambda} \frac{\mathrm{e}^{\lambda(k+1) r}-1}{\mathrm{e}^{\lambda r}-1}
\end{aligned}
$$

which ends the proof.

\section{B Light-tailed calculations for $k>2$}

Here, let us prove that, for $k>2$,

$$
\varphi^{(k)}(\alpha, \beta)=\frac{g(\alpha, \beta)^{k} \widehat{F}[\alpha]^{k-1} \widehat{G}[\alpha]}{1-k(\alpha, \beta) \widehat{F}[\alpha]\left[1-(g(\alpha, \beta) \widehat{F}[\alpha])^{k}\right] /[1-g(\alpha, \beta) \widehat{F}[\alpha]]},
$$

where

$$
\begin{aligned}
g(\alpha, \beta) & =\frac{\lambda}{\lambda+c \alpha-\beta} \mathrm{e}^{(\beta-c \alpha-\lambda) r}, \\
k(\alpha, \beta) & =\frac{\lambda}{\lambda+c \alpha-\beta}\left(1-\mathrm{e}^{(\beta-c \alpha-\lambda) r}\right),
\end{aligned}
$$


and $\varphi^{(k)}(\alpha, \beta)=\mathbb{E}\left[\mathrm{e}^{\alpha S(\omega)} \mathrm{e}^{\beta \omega}\right]$ in the $k$-case. We use the same notation as in Section 5. A $(k>2)$-cycle begins as a $(k=2)$-cycle with some short interarrival times, each followed by an $F$-claim with contribution to $\varphi^{(k)}(\alpha, \beta)$ equals to

$$
\frac{p_{+}}{1-p_{-} \widehat{F}[\alpha] \widehat{A}^{-}[\beta-c \alpha]},
$$

followed by a long interarrival time and an $F$-claim which gives a contribution of

$$
\widehat{F}[\alpha] \widehat{A}^{+}[\beta-c \alpha] .
$$

After that, there are $k$ possible paths. One path is constituted with $k-1$ long interarrival times. The first $k-2$ times are followed by an $F$-claim and the last one by a $G$-claim and so the cycle terminates. This path has a probability $p_{+}{ }^{k-1}$ to occur and gives a contribution of

$$
\left(p_{+} A^{+}[\beta-c \alpha]\right)^{k-1} \widehat{F}[\alpha]^{k-2} \widehat{G}[\alpha] .
$$

For $0 \leq i \leq k-2$, the $i$-path is constituted with $i$ long interarrival times and one short time, each followed by an $F$-claim, then the cycle starts afresh after that. The $i$-path occurs with probability $p_{+}{ }^{i} p_{-}$and gives a contribution of

$$
p_{+}{ }^{i} p_{-} \widehat{F}[\alpha]^{i+1} A^{+}[\beta-c \alpha]^{i} A^{-}[\beta-c \alpha] \varphi^{(k)}(\alpha, \beta) .
$$

Putting things together gives

$$
\begin{gathered}
\varphi^{(k)}(\alpha, \beta)=\frac{p_{+}}{1-p_{-} \widehat{F}[\alpha] \widehat{A}^{-}[\beta-c \alpha]} F[\alpha] \widehat{A}^{+}[\beta-c \alpha] \times\left(\left(p_{+} A^{+}[\beta-c \alpha]\right)^{k-1} \widehat{F}[\alpha]^{k-2} \widehat{G}[\alpha]\right. \\
\left.+\sum_{i=0}^{k-2} p_{+}{ }^{i} p_{-} \widehat{F}[\alpha]^{i+1} A^{+}[\beta-c \alpha]^{i} A^{-}[\beta-c \alpha] \varphi^{(k)}(\alpha, \beta)\right),
\end{gathered}
$$

which ends the proof.

\section{Proof of (6.3)-(6.7)}

The expression (6.3) for $h^{\theta}(-, 0)$ is just Proposition 5.1.

To compute $h^{\theta}(i)$ for all $i$, we first introduce some notation, with $V$ an exponential random variable with rate $\lambda$ :

$$
\begin{aligned}
p_{(a)+} & =\mathbb{P}(V>r-a)=\mathrm{e}^{-\lambda(r-a)}, \\
p_{(a)-} & =\mathbb{P}(V \leq r-a)=1-\mathrm{e}^{-\lambda(r-a)}, \\
\widehat{A}[\alpha] & =\mathbb{E}\left[\mathrm{e}^{\alpha V}\right]=\frac{\lambda}{\lambda+\alpha}, \\
\widehat{A}^{(a)+}[\alpha] & =\mathbb{E}\left[\mathrm{e}^{\alpha V} \mid V>r-a\right]=\frac{\lambda}{\lambda-\alpha} \mathrm{e}^{\alpha(r-a)}, \\
\widehat{A}^{(a)-}[\alpha] & =\mathbb{E}\left[\mathrm{e}^{\alpha V} \mid V \leq r-a\right]=\frac{\lambda}{\lambda-\alpha}\left(\frac{1-\mathrm{e}^{-(\lambda-\alpha)(r-a)}}{1-\mathrm{e}^{-\lambda(r-a)}}\right),
\end{aligned}
$$


and we are using $p_{+}, p_{-}, A^{+}[\alpha]$ and $A^{-}[\alpha]$ defined in Sections 3,5 .

The easiest case is the $(+,+)$-one. Actually, after an exponential time, a $G$-claim occurs which ends the cycle, so

$$
\begin{aligned}
h^{\theta}(+,+) & =\mathbb{E}_{(+,+)} \mathrm{e}^{\theta S\left(\omega_{1}\right)-\kappa(\theta) \omega_{1}}=\mathbb{E} \mathrm{e}^{-(c \theta+\kappa(\theta)) V} \mathrm{e}^{U_{G}} \\
& =\widehat{A}[-c \theta-\kappa(\theta)] \widehat{G}[\theta]=\frac{\lambda}{\lambda+c \theta+\kappa(\theta)} \widehat{G}[\theta] .
\end{aligned}
$$

For the $(+, a)$-case, two cases have to be considered. If the exponential time before the first claim is larger than $r-a$ then a $G$-claim occurs, otherwise, an $F$-claim occurs and the state becomes $(-, 0)$ :

$$
\begin{aligned}
h^{\theta}(+, a) & =\mathbb{E}_{(+, a)} \mathrm{e}^{\theta S\left(\omega_{1}\right)-\kappa(\theta) \omega_{1}}, \\
& =p_{(a)-} \widehat{A}^{(a)-}[-c \theta-\kappa(\theta)] \widehat{F}[\theta] h^{\theta}(-, 0)+p_{(a)+} \widehat{A}^{(a)+}[-c \theta-\kappa(\theta)] \widehat{G}[\theta] \\
& =\frac{k^{(a)}(\theta,-\kappa(\theta)) g(\theta,-\kappa(\theta))^{2} \widehat{F}[\theta]^{2} \widehat{G}[\theta]}{1-k(\theta,-\kappa(\theta)) \widehat{F}[\theta](1+g(\theta,-\kappa(\theta)) \widehat{F}[\theta])}+g^{(a)}(\theta,-\kappa(\theta)) \widehat{G}[\theta] .
\end{aligned}
$$

The particular case $(+, 0)$ gives

$$
\begin{aligned}
h^{\theta}(+, 0) & =k^{(0)}(\theta,-\kappa(\theta)) h^{\theta}(-, 0)+g^{(0)}(\theta,-\kappa(\theta)) \\
& =\frac{g(\theta,-\kappa(\theta)) \widehat{G}[\theta](1-k(\theta,-\kappa(\theta)) \widehat{F}[\theta])}{1-k(\theta,-\kappa(\theta)) \widehat{F}[\theta](1+g(\theta,-\kappa(\theta)) \widehat{F}[\theta])} .
\end{aligned}
$$

The $(-,+)$-case can be easily solved using the $(+, 0)$ one. Actually, after an exponential time, an $F$-claim occurs and the state becomes $(+, 0)$ :

$$
\begin{aligned}
h^{\theta}(-,+) & =\mathbb{E}_{(-,+)} \mathrm{e}^{\theta S\left(\omega_{1}\right)-\kappa(\theta) \omega}=\widehat{A}[-c \theta-\kappa(\theta)] \widehat{F}[\theta] h^{\theta}(+, 0) \\
& =\frac{\lambda}{\lambda+c \theta+\kappa(\theta)} \frac{g(\theta,-\kappa(\theta)) \widehat{F}[\theta] \widehat{G}[\theta](1-k(\theta,-\kappa(\theta)) \widehat{F}[\theta])}{1-k(\theta,-\kappa(\theta)) \widehat{F}[\theta](1+g(\theta,-\kappa(\theta)) \widehat{F}[\theta])} .
\end{aligned}
$$

We solve the $(-, a)$-case using both $(+, 0)$ and $(-, 0)$ ones. If the exponential time before the first claim is larger than $r-a$, then an $F$-claim occurs and the state becomes $(+, 0)$, otherwise, an $F$-claim occurs and the state becomes $(-, 0)$ :

$$
\begin{aligned}
h^{\theta}(-, a)= & \mathbb{E}_{(-, a)} \mathrm{e}^{\theta S\left(\omega_{1}\right)-\kappa(\theta) \omega_{1}}, \\
= & p_{(a)-} \widehat{A}^{(a)-}[-c \theta-\kappa(\theta)] \widehat{F}[\theta] h^{\theta}(-, 0) \\
+ & p_{(a)+} \widehat{A}^{(a)+}[-c \theta-\kappa(\theta)] \widehat{F}[\theta] h^{\theta}(+, 0), \\
= & \frac{g(\theta,-\kappa(\theta)) \widehat{F}[\theta] \widehat{G}[\theta]}{1-k(\theta,-\kappa(\theta)) \widehat{F}[\theta](1+g(\theta,-\kappa(\theta)) \widehat{F}[\theta])}\left\{k^{(a)}(\theta,-\kappa(\theta)) g(\theta,-\kappa(\theta)) \widehat{F}[\theta]\right. \\
& \left.\quad+g^{(a)}(\theta,-\kappa(\theta)) \widehat{G}[\theta](1-k(\theta,-\kappa(\theta)) \widehat{F}[\theta])\right\} .
\end{aligned}
$$




\section{A counterexample}

We give here an example of random variables $X, Y$ such that $X$ is subexponential and $Y$ light-tailed, but the tail of $X-Y$ is lighter than the tail of $X$ (of course, such r.v.'s have to be dependent).

We take $X$ with tail $\bar{F}(x)=\mathrm{e}^{-x / \log x}, x>$ e. Then the density and failure rates are

$$
f(x)=\left[\frac{1}{\log x}-\frac{1}{\log ^{2} x}\right] \mathrm{e}^{-x / \log x}, \text { resp. } \lambda(x)=\frac{f(x)}{\bar{F}(x)}=\frac{1}{\log x}-\frac{1}{\log ^{2} x} .
$$

The integral to be inspected for Pitman's criterion ([5, p. 299]) is

$$
\int_{\mathrm{e}}^{\infty} \mathrm{e}^{x \lambda(x)} f(x) \mathrm{d} x=\int_{\mathrm{e}}^{\infty} \mathrm{e}^{-x / \log ^{2} x}\left[\frac{1}{\log x}-\frac{1}{\log ^{2} x}\right] \mathrm{d} x,
$$

which is finite so that $X$ is subexponential.

Let $Y=\varphi(X)=X / \log X$ and note that whenever $z<\varphi^{-1}(x)$, i.e. $\varphi(z)<$ $x$, then

$$
\mathbb{P}(Y>x)=\mathbb{P}\left(X>\varphi^{-1}(x)\right) \leq \mathbb{P}(X>z) .
$$

Taking $z=z(x)=x \log x$, we have

$$
\varphi(z)=\frac{x \log x}{\log x+\log \log x}<x .
$$

and thus

$$
\mathbb{P}(Y>x) \leq \mathbb{P}(X>z)=\exp \{-x \log x /[\log x+\log \log x]\}<\mathrm{e}^{-x / 2}
$$

for all large $x$. Thus $Y$ is light-tailed.

Let $0<b<a<1$ and note that $X-Y=\psi(X)=X(1-1 / \log X)$. Taking $z=z(x)=x+a x / \log x$, we get

$$
\psi(z)=(x+a x / \log x)\left[1-\frac{1}{\log x[1+\log (1+a / \log x) / \log x]}\right]<x
$$

for large $x$ (using $a<1$ ), and thus as above,

$$
\mathbb{P}(X-Y>x) \leq \mathbb{P}(X>z)=\mathrm{e}^{-z / \log z} .
$$

Now

$$
\begin{aligned}
\frac{z}{\log z} & =\frac{x+a x / \log x}{\log x[1+\log (1+a / \log x) / \log x]} \\
& \geq \frac{x+a x / \log x}{\log x}[1-[1+\log (1+a / \log x) / \log x]] \\
& \geq \frac{x}{\log x}+\frac{b x}{\log ^{2} x}
\end{aligned}
$$

for all large $x$ (using $(1+v)^{-1}>1-v$ in the second step). Thus

$$
\mathbb{P}(X-Y>x) \leq \mathbb{P}(X>x) \mathrm{o}(1),
$$

and the counterexample is complete. 


\section{References}

[1] H. Albrecher \& S. Asmussen (2006) Ruin probabilities and aggregate claims distributions for shot noise Cox processes. Scand. Actuar. J. 2006, 86-110.

[2] H. Albrecher \& O. Boxma (2004) A ruin model with dependence between claim sizes and claim intervals. Insurance: Math. Econom. 35, 245-254.

[3] V. Asimit \& A. Badescu (2010) Extremes on the discounted aggregate claims in a time dependent risk model. Scand. Actuar. J. 2010, 93-104.

[4] S. Asmussen (2003) Applied Probability and Queues (2nd ed.). Springer-Verlag.

[5] S. Asmussen \& H. Albrecher (2010) Ruin Probabilities (2nd ed.). World Scientific.

[6] S. Asmussen \& C. Klüppelberg (1996) Large deviations results for subexponential tails, with applications to insurance risk Stoch. Proc. Appl. 64, 103-125

[7] S. Asmussen, H. Schmidli \& V. Schmidt (1999) Tail approximations for nonstandard risk and queueing processes with subexponential tails. Adv. Appl. Probab. 31, 422-447.

[8] G. Balkema \& L. de Haan (1974) Residual life-time at great age. Ann. Probab. 2, 792-804.

[9] R. Biard, C. Lefevre, C. Loisel \& H.Nagaraja (2010) Asymptotic finite-time ruin probabilities for a class of path-dependent heavy-tailed claim amounts using Poisson spacings. Appl. Stoch. Models Bus. Ind. (to appear).

[10] M. Boudreault, H. Cossette, D. Landriault \& E. Marceau (2006) On a risk model with dependence between interclaim arrivals and claim sizes. Scand. Actuar. J. 2006, 265-285.

[11] D.J. Daley (ed., 2001) Probability, Statistics and Seismology. A Festschrift for David Vere-Jones. J. Appl. Probab., Special Volume 38A.

[12] N.G. Duffield \& N. O'Connell (1995) Large deviations and overflow probabilities for the general single-server queue, with applications. Math. Proc. Camb. Philos. Soc. 118, 363-374.

[13] P. Embrechts, C. Klüppelberg \& T. Mikosch (1997) Modelling Extreme Events for Insurance and Finance. Springer-Verlag.

[14] S. Foss, D. Korshunov \& S. Zachary (2009) An Introduction to Heavy-tailed and Subexponential Distributions. Oberwolfach Preprints (OWP) 2009-13.

[15] S. Foss, Z. Palmowski \& S. Zachary (2005) The probability of exceeding a high boundary on a random time interval for a heavy-tailed random walk. Ann. Appl. Probab. 15, 1936-1957.

[16] S. Foss, T. Konstantopoulos \& S. Zachary (2007) Discrete and continuous time modulated random walks with heay-tailed increments. J. Theor. Probab. 20, 581612 .

[17] P.W. Glynn \& W. Whitt (1994) Logarithmic asymptotics for steady-state tail probabilities in a single-server queue. In Studies in Applied Probability (J. Galambos \& J. Gani, eds.). J. Appl. Probab. 31A, 131-156.

[18] P. Jelenković, P. Momcilović \& B. Zwart (2004) Reduced load equivalence under subexponentiality. QUESTA 46, 97-112. 
[19] J. Li, Q. Tang \& R. Wu (2010) Subexponential tails of discounted aggregate claims in a time-dependent renewal risk model. Adv. in Appl. Probab. 42, 11261146.

[20] S. Resnick (1987) Extreme Values, Regular Variation, and Point Processes. Springer-Verlag.

[21] H. Schmidli (1997) An extension to the renewal theorem and an application in risk theory. Ann. Appl. Probab. 7, 121-133. 